\title{
Fractal Management Systems for Extended, Holonic Enterprises
}

\author{
Pierluigi Assogna \\ Theorematica $\operatorname{SpA}$ \\ Via Simone Martini 143-00142 Roma \\ p.assogna@theorematica.it
}

\begin{abstract}
The complexity and the extension of the production environment are increasing at a fastening pace. This ever-increasing complexity requires new and more flexible Management Systems and decision supports that go above and beyond ERP's. Any information system designed and developed to support a manufacturing enterprise has as a (sometimes implicit) first step: the modeling of the enterprise. Four metaphors are briefly explored, in relation to the increasing complexity of the environment to be modeled, and to the parallel increasing flexibility of the resulting models:

- The econometric model

- The hydraulic model

- The cybernetic model

- The chain value (Porter)

In these last years a new enterprise organization called holonic or cellular has been defined. It is embodied by a multilevel network of holons, a network that has a high degree of flexibility, because it can easily restructure itself depending on the circumstances: in fact each holon has the capability of surviving even if detached by any network, and the ability to search for a new network to integrate into. The holonic structure has a fractal nature, so that each holon is in turn made of holons, and the single persons cooperating in the enterprise represent the lower level. This structure is the best one for enduring the chaotic evolution of the manufacturing environment. The paper concludes with the proposal of a modeling technique to be used when designing the Management System for a manufacturing organization.
\end{abstract}

Key words: Manufacturing, Holon, Fractal, Modeling

The original version of this chapter was revised: The copyright line was incorrect. This has been corrected. The Erratum to this chapter is available at DOI: 10.1007/978-0-387-35492-7_50 


\section{Foreword}

Manufacturing Organizations are complex systems in complex environments.

Complex systems have the tendency (not completely explained) to drift towards places and situations where life is dangerous. They seem to be attracted by precipices.

This specific zone of the possible states of the system is called "the edge of chaos".

A Manufacturing Organization lives in turbulent environments, where turbulence is ever increasing. In order to survive and prosper it has to be able to anticipate troubles (cataclysms in complexity jargon) and take advantage as much as possible of relaxed periods to prepare for cataclysms.

Globalization is a typical tidal force that very often triggers a cataclysm in traditional manufacturing organizations, mainly because of the requirement of flexibility.

Flexibility has been the name of the game for some time now, but it is important to define what and where has to be flexible within the organization.

\section{The harsh requirements of complex environments}

Complex systems have a characteristic structure, which is that of fractals.

Fractals are mathematical formulas that, fed to an engine that builds geometric loci on a computer screen, come up with often spectacular images that look like vortices, complex spirals, clouds, embroideries.

Fractals (or to be more precise their computer renderings) have the characteristic that their main visual aspect (be it a vortex, a pattern of spikes, or any) repeats itself at different scale all the way down to infinity.

Real life examples of fractals are clouds, coastal shapes, water whirls, tree branches, river deltas, and others.

All these are complex systems, so that we can say that a complex system is always a fractal, that means that it is composed of sub-systems and subsub-systems and so on, all isomorphic.

Many different complex systems inter-related make a complex environment: at different levels different components (systems) inter-react in a very complex network of relations and feedbacks.

Because of this multi-level lattice of relations, everything is in constant chance of change. 
This change, when it happens, has an interesting characteristic: it is an evolution, in the sense that the systems tend to become (at each major shift of their shape) more complex, with a richer structure. Systems that do not have this capacity of reorganizing at a higher structure complexity do not survive for long in such an environment.

From this viewpoint a complex environment is very demanding: either you are prepared, every now and then, to re-organize, or you perish.

Of course there are events that can completely destroy this sort of environments, but if we take as an example life on earth, you need a cosmic cataclysm to do the job.

This picture is very different from the mechanistic systems of reductionism ages (La Place's universe), where every sub-system had a precise configuration, and where its only possible change was from functioning to non-functioning.

No matter how accurately a system builds to itself a cozy niche, sooner or later the niche disappears, leaving the system with the old dilemma: evolve or perish.

\section{How to keep evolving}

Fortunately, this changing is not continuous, but discrete.

Complex systems do not evolve continuously, but in a discrete way: this is what S. Jay Gould calls "punctuated evolution". This happens when multiple systems co-evolve in a common environment, that is the typical situation with living organisms or social systems: each system remains in a quasi-static situation for some time, than is caught in some sort of cataclysm (usually short) and than again can rest for some more time. The lengths of calm periods are unequal, and the size of each cataclysm is variable.

There are two main reasons for this complex behavior.

The first comes from the natural tendency of every system to resist to changes: it is called homeostasis. A system tends to keep its configuration despite any external influence: it exercises all its mechanisms in order to absorb the disturbance.

Of course there is a limit to the capability of staying in shape under any amount of pushes and pulls. Once the threshold is reached, the system all of a sudden finds itself at a bifurcation: either it perishes, or it finds (fast) a new configuration that lets it survive under new conditions.

Every time a system pertaining to a complex environment passes through such a cataclysm, it sends shock waves all around, and this maybe triggers the turmoil of another system. Because of this interdependency, each system goes through the punctuated evolution. 
The second reason of the discreteness of the size of cataclysms comes from the fractal-like multi-level structure of complex systems.

The major shifts (or evolution steps) that happen in a system involve one of the levels of its structure, if not the entire system. Depending on the level that participates in this cataclysm, its magnitude follows a quantum-like pattern of discreteness.

A given level enters a cataclysm when its direct components become unstable (generally because of exogenous disturbances), and as seen, before giving up it resists for some time.

Now, coming back to our Manufacturing Organization, it needs a mechanism that helps effectively in taking the opportunity, every time a blow strikes, to re-organize on a better stand.

\section{Knowledge can help}

The only basic mean that can help survival is knowledge. Only by knowing as much as possible about itself and its environment a system can survive and prosper.

In the case of a manufacturing organization the main tool for knowledge management is of course its Information System, that in recent years means its Enterprise Resource Planning (ERP) system.

Now, if we take a close look at the best sellers in this arena, we can see that all of them have as common and noble father the Material Requirements Planning (MRP I). This technique was devised in the 60's, when a manufacturing organization was supposed to stay stable within a stable environment. You can tell this by analyzing the way that these systems treat changes (be these engineering or material source, or whatever): changes are a nuisance, something that force procedures to amend situations, cancel transactions, revert flows, etc.

In a word, traditional ERP's are geared to a quasi-static world

If we take an integrated system such as an ERP, we can see it as a model of the organization and of its context (suppliers, customers, and partners). What is getting more and more inadequate is exactly this embedded model.

Modeling an organization means formalizing the relevant knowledge, and as seen, knowledge is the recipe for survival in complex situations.

Does this mean that we have to throw away ERP's? Definitely no, but we need probably to chop them (a situation similar to the broom of the "Apprentice Sorcerer") into interconnected mini-ERP's, as described later on. 


\section{Modeling metaphors}

Let's start from the beginning, that is modeling.

When you start this exercise, you have in mind (explicitly or implicitly) a metaphor, a meta-model that provides the syntax of the modeling.

The resulting models essentially have to describe the interactions of Actors, Objects, Processes, and the granularity of this representation is very important for our talk.

In order to guide the modeling of the Objects, Actors, Processes of a manufacturing enterprise, different meta-models have been historically utilized, depending on the inclination of the domain experts, on the strengths and weaknesses of the meta-models, and on the dominating business paradigm of the day.

A meta-model is a metaphor, and as such is used to provide analogues for all the different aspects of the production environment that needs to be modeled. In order to model some specific aspects, the analogy needs to be stretched to a limit where the power of evocation, that is the main advantage of using metaphors, thins out; for this reason none of the typical metaphors is perfect in each and every respect.

We can briefly analyze four different metaphors, keeping in mind this granularity, and see for each what are the pro's and con's, in terms of:

- Degree of coverage of activities

- Formalism

- Evocation power

- Modularity

- Degree of complexity that can be represented

\section{The econometric metaphor}

It is based on the Cournot duopoly model. The adopted formalism is mathematically concise.

It is geared essentially to the general input and output of the organization, in terms of money.

For this metaphor the enterprise is basically seen as a monolith.

\section{The hydraulic metaphor}

It is a simplified application of Forrester's System Dynamics: the organization is seen as a whole system, where each part is "mechanically" connected to some other parts.

In this metaphor water is used to represent the connections because of its non-deformability, and because it does not change with time.

Mathematical formulas regulate the exchanges between these parts. 


\section{The cybernetic metaphor}

Introduced by Stafford Beer [1], utilizes the organism and its mechanisms as the metaphoric analogy.

A very important step of this methodology is the analysis of the number of different states that a sub-system can reach. An axiom is derived from this analysis, i. e. that you cannot control effectively a system with $n$ possible states with another system with $m$ possible states where $m<n$.

Said in other words, the "knowledge" of the controller must be greater than that of the controlled.

Feedback and feed-forward circuits are very much components of the models created with this methodology.

\section{The chain value (Porter) metaphor}

Introduced by $\mathrm{M}$. E. Porter [2], gives an economic value to every process (relation) between a system and its context.

There are 5 main set of activities that participate directly in the process, and 4 that support the process.

The scheme of this representation is the following:

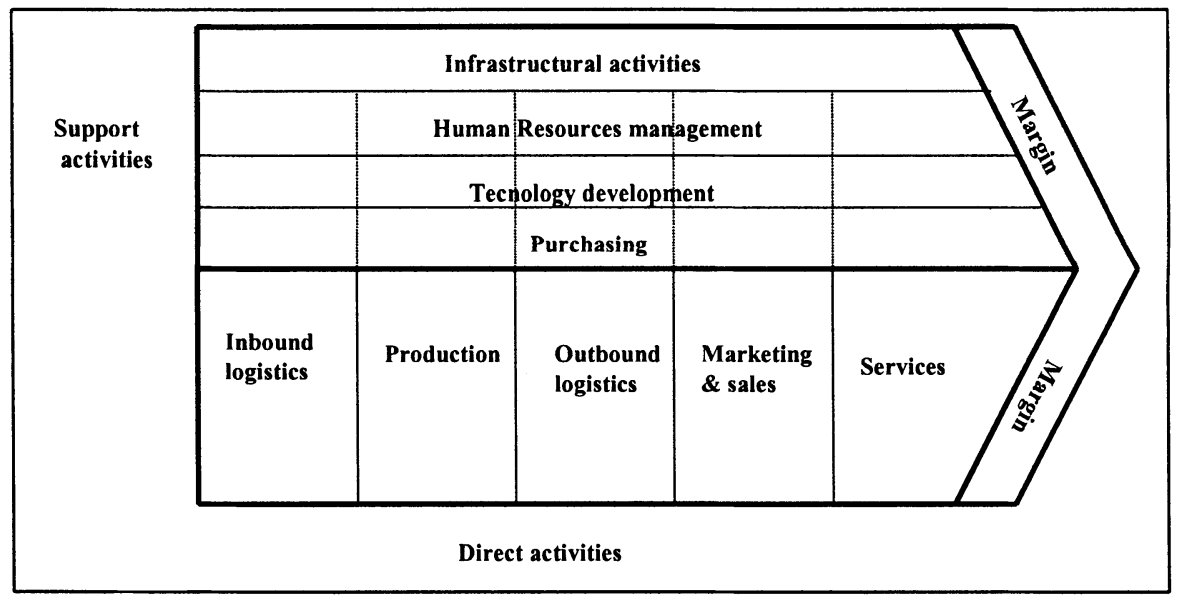

The Value Chain

This scheme can be applied both to the enterprise and to its components, provided that you can identify for each the described activity sets. 
The resulting table of strengths and weaknesses is more or less the following:

\begin{tabular}{|c|l|l|l|c|}
\hline \multirow{2}{*}{} & \multicolumn{4}{|c|}{ Metaphors } \\
\cline { 2 - 5 } & Econometric & \multicolumn{1}{|c|}{ hydraulic } & \multicolumn{1}{|c|}{ Value Chain } & Cybernetic \\
\hline Degree of coverage & Good & $\begin{array}{l}\text { Good for marketing } \\
\text { and administration }\end{array}$ & $\begin{array}{l}\text { Good for } \\
\text { logistics and } \\
\text { administration }\end{array}$ & Good \\
\hline Formalism & $\begin{array}{l}\text { Good for } \\
\text { synthesis, not } \\
\text { for analysis }\end{array}$ & Good & Good & Average \\
\hline $\begin{array}{c}\text { Evocation } \\
\text { power }\end{array}$ & Scarce & Average & Good & Good \\
\hline Modularity & Scarce & Average & Average & Good \\
\hline Complexity & Scarce & Scarce & Average & Good \\
\hline
\end{tabular}

The table indicates that from the viewpoint of the complexity that can be described, and modularity of the models that you generate, the value chain and the cybernetic meta-models are the best ones.

There is also a trend, from the econometric to the value chain, from a monolithic to a composite view of the organization.

The system, as seen with the hydraulic meta-model, is mechanical, in the sense that the relations between the components are rigid and linear.

\section{The Holonic Fractal Enterprise}

The term holon was introduced by Arthur Koestler [3] and indicates a (sub)system capable of functioning alone, and at the same time prone to (and capable of working in) cooperation with other holons.

A holon can live by itself, but is better off when integrated into a system, a holonic structure.

It can well represent an organization component, at any level of a complex structure, that even if perfectly integrated in the business activity, is able of detach itself from the structure and attach to another one, if and when the circumstances should require this move.

And if the structure is all made of holons, it is not disrupted by migrations of this sort.

A holonic structure is normally made of holons of different kinds, and the more diverse the holons, the higher the repertoire of behaviors of the structure, and thus its survival capability. 
Each holon has a core competence that justifies its presence in the structure, plus "a little bit" of all other competences that are used to negotiate its participation.

An holonic enterprise is made of holons, in turn made of holons, and can participate in higher level structures. As each holon has to have a common set of negotiating (or interface) capabilities, we can see this enterprise as a fractal enterprise, with isomorphic components.

This sort of enterprise is better equipped to afford the vagaries and blows of the global market, because of its inherent flexibility, and when a new technology changes some aspects of the stage, this network can re-organize itself, and the holons no more fit for the new picture migrate to other structures, and are replaced by new ones.

This flexibility and robustness involve the knowledge that each single holon has of its internal workings (that is its model) and of its context, that cannot be represented by the components it is related to.

It is exactly this the main difference between a holon and a traditional component: the former must be constantly aware of as much world as possible, while the latter just needs to know its surrounding. The same knowledge requirement applies to the supporting software of the holon

From the viewpoint of the modeling, this structure can be easily modeled both by the cybernetic and the value-chain, as each holon can be seen as a "mini-enterprise".

It could even be possible to integrate, in one final model, holons modeled with different methodologies, in order to exploit the different representation strengths of the metaphors for different sectors of an organization.

\section{The fractal ERP}

Going back to the fact that the ERP system is the working model of a manufacturing organization, a holonic enterprise needs an ERP structured as a fractal.

What is needed is an ERP made of mini-ERP's, each one made of microERP's, on many levels.

It may sound bizarre, but in a sense we can see this structure in the material logistics planning and control of traditional ERP's: in fact these functions are normally embodied in long, medium, short term cycles that have recursive logic.

Even in the level-by-level planning of MRP this fractal nature is present. 
But traditional ERP's come short of this granularity target: their implicit model is too much centralized. They need to be re-architected in fractal-like structures.

If we remember the knowledge requirement, it is not enough to take a traditional ERP module as the management mechanism of each holon.

Now the image of the broom of the Sorcerer, that chopped into minibrooms performs its task more efficiently, becomes clear.

As a conclusion we suggest the following procedure, in order to develop a holonic Management System for a manufacturing organization:

1. Analyze the organization as made of a multi level structure of holons, finding for each component all the activities of an entire enterprise, even if only in germ.

2. Choose for each of these holons the most effective modeling metaphor, taking into account the "evocative" strength of the analogy that in this exercise is a very important asset. The resulting model has anyway to have Actors, Objects and Processes: the Object Oriented paradigm is too effective not to be adopted.

3. Assemble the holon at the enterprise level by putting in relation all the components.

4. At this point it is possible to find modules of ERP's that fit the picture at different levels, and supplement these with sort of "wrappings", that provide the whole sets of functionality that make the holon capable of staying autonomous.

\section{Bibliography}

[1] The managerial cybernetics of organization (Allen Lane The Penguin Press Ed., 1972).

[2] Competitive Advantage: Creating and Sustaining Superior Performance(Free Press, M. E. Porter, 1985)

[3] Beyond atomism and holism: the concept of the holon. (A. Koestler and J.R. Smythies eds. Beyond reductionism. Hutchinson, London). 